\title{
EVALUATING CHANGES IN THE DRIVING BEHAVIOR OF YOUNG DRIVERS A FEW YEARS AFTER LICENSURE USING IN-VEHICLE DATA RECORDERS
}

\author{
Gila Albert ${ }^{1}$, Oren Musicant ${ }^{2}$, Tsippy Lotan $^{3}$, Tomer Toledo ${ }^{4}, \&$ Einat Grimberg ${ }^{3}$ \\ ${ }^{1}$ Holon Institute of Technology (HIT), Holon, Israel \\ ${ }^{2}$ Tel-Aviv University, Tel-Aviv, Israel \\ ${ }^{3}$ Or Yarok, Hod Hasharon, Israel \\ ${ }^{4}$ Technion-Israel Institute of Technology, Haifa, Israel \\ Email: gila.albert1@gmail.com
}

\begin{abstract}
Summary: This paper aims to evaluate how young drivers drive a few years after licensure. Driving behavior in the fourth year of driving is compared to that of the first year, based on data from In-Vehicle Data Recorders (IVDR). Young drivers' cars were equipped with the same IVDR systems in both study periods. The comparison revealed that, in general, driving patterns did not change significantly. The difference in risky behaviour between weekdays and weekends was more prominent in the fourth year than in the first year. In addition, an interesting improvement occurred at the end of the fourth-year study period. The analysis results obtained should also be considered an example of the potential of what may be done with this kind of data.
\end{abstract}

\section{INTRODUCTION}

Young drivers in Israel, similar to other places around the globe, are involved in car crashes more than any other age group. Typically, the definition of a "young driver" in Israel includes new drivers between the ages of 17-24. However, safety literature pays primary attention to young drivers aged 17-18, rather than young drivers aged 19-24 (Doherty et al.,1998; Lotan and Toledo, 2007; Williams, 2003).

This paper is based on a study done in Israel as part of the PROLOGUE project conducted within the European Union's Seventh Framework Programme (EU FP7). The study aims to evaluate how young drivers drive three to four years after licensure. Their driving behavior is compared to how they drove in the first year after licensure at the age of 17-18. Driving behavior and patterns throughout the year following licensure was explored in a previous study conducted between the years 2006-2008 with 120 novice young drivers in Israel. For a detailed description of this study and its results, see Lotan and Toledo (2007), Toledo et al. (2008) and Prato et al. (2010).

In both studies, the evaluation of driving behavior and patterns was performed using IVDR. This advanced recording equipment was installed in the drivers' cars and, consequently, all trips made by the vehicle were monitored and information regarding trip characteristics and safety levels were stored and conveyed to participants. The application of IVDR to continuously monitor driving patterns and behavior, not only during a crash event, has been recognized in various studies (Toledo et al., 2008; Dingus et al., 2006).

The fourth-year study presented in this paper is, in many ways, a follow-up study to the first-year study. To the best of our knowledge, this is the first time that a follow-up study with naturalistic data has been conducted in this context. 


\section{METHODOLOGY}

GreenRoad IVDR systems were re-installed in the cars of some of the young drivers who participated in the first-year study. The participants drove their own cars, or their family cars, which were equipped with IVDR, on their regular trips. All of these trips were monitored. Participants were asked to identify themselves using a magnetic key.

The participants were recruited from the participants' pool of the first-year study. Those who agreed to participate again were screened for car availability and sufficient driving experience. The response was quick and mostly positive. Eventually, 32 young drivers participated in this study, after signing an informed consent form. Out of these, $21(66 \%)$ are males and $11(34 \%)$ are females. Their average age at the time the study began was $20.5 \pm 0.5$ years. The majority of subjects (75\%) performed regular military service during most of the period in which the study took place (in Israel, young people aged 18 are required to perform at least 2-3 years of national military service). Therefore, their use of passenger cars was assumed not to be intensive. Generally, their car travel occurred when they were off-duty. On average, subjects got their driving license $40.0 \pm 6.6$ months prior to the re-installation of the IVDR.

The GreenRoad IVDR used in both studies is able to identify the occurrence of undesirable driving events such as hard braking, accelerating, sharp turning, and swift lane changes, and to report these events to the driver in real time. It also transmits this data to a server that stores the information in a database. A web application supplies drivers with aggregated information about the occurrence of these driving events. In addition, the IVDR provides the start time and end time of the trip, which is used to calculate the driving time.

The events rate is given by the ratio between the safety events (defined as: braking, accelerating, turning, lane handling and speeding) and total driving time. This events rate was used as an index for comparison between the first and fourth year. A mixed effect Poisson-lognormal regression model was used to estimate the log events rate for each driver. In addition, the events rate may change depending on the time of day and day of week (Musicant et al.,2010), so these temporal explanatory variables were also considered in our analysis.

In some of our previous studies, the usefulness of providing feedback from technology was explored, and it was found to be linked to a decrease in the events rate (Musicant and Lampel 2010; Toledo et al., 2008). The fourth-year study was therefore designed to test the effect of feedback as part of the follow-up.

The data collection period for the fourth-year study lasted 8 months. The experimental design included three stages. The first stage ("no feedback") started immediately after the installation and lasted about 2.5 months. In this stage, the IVDR was installed in the vehicle, but the participants did not receive any feedback from it. In the feedback stage that followed, participants received feedback via web reports and the in-vehicle display. This stage lasted about 3.5 months. Finally, a two month cool-off stage was administered, in which the participants continued to drive with the IVDR but did not receive any feedback. The data obtained through the IVDR system was compared to the data collected in the first-year study. 


\section{RESULTS}

\section{Summary Statistics}

Table 1 presents general statistics of the trips the participants undertook in the two studies. It should be noted that, as novice drivers, the participants took 1.55 trips per day, but as more experienced drivers, they took only 1.02 trips per day. This may be explained, as noted earlier, by the limitations imposed by their military service.

Table 1. Characteristics of the trips undertaken by the young drivers

\begin{tabular}{lcccccccc}
\hline & \multicolumn{3}{c}{ First-year study } & & \multicolumn{3}{c}{ Fourth-year study } \\
\cline { 2 - 3 } \cline { 7 - 8 } & $\begin{array}{c}\text { Accom- } \\
\text { panied }\end{array}$ & $\begin{array}{c}\text { No } \\
\text { Feedback }\end{array}$ & Feedback & & $\begin{array}{c}\text { No } \\
\text { Feedback }\end{array}$ & Feedback & Cool-off \\
\hline Number of trips & 1,267 & 2,313 & 11,211 & & 1,859 & 3,050 & 1,565 \\
Trip duration (min) & 23.6 & 20.7 & 20.8 & & 27.3 & 23.2 & 23.0 \\
average (SD) & $(21.5)$ & $(18.5)$ & $(18.9)$ & & $(22.8)$ & $(26.1)$ & $(22.1)$ \\
\hline
\end{tabular}

\section{Comparison of Events Rates}

The estimated events rate for the various stages of the studies are presented in Figure 1. The width of each box is proportional to the number of trips in the specific stage. In the first-year study, the accompanied driving stage is clearly characterized by the lowest events rate, which seems plausible and in line with the impact of this stage on safety (Lotan and Toledo, 2007). This effect is prominent enough to be detected by the statistical analysis, even with the relatively small sample size used in our study. Repeated measures ANOVA $\left(\mathrm{F}_{(2,44)}=16.50, \mathrm{p}\right.$.

value $<0.001)$, followed by the Tukey post-hoc test $(\alpha=0.05)$, did reveal that the events rate in the accompanied driving stage is different from the other two stages. A small decline in events rates is also observed in the passage from the no feedback stage to the feedback stage. However, this effect was not statistically significant.

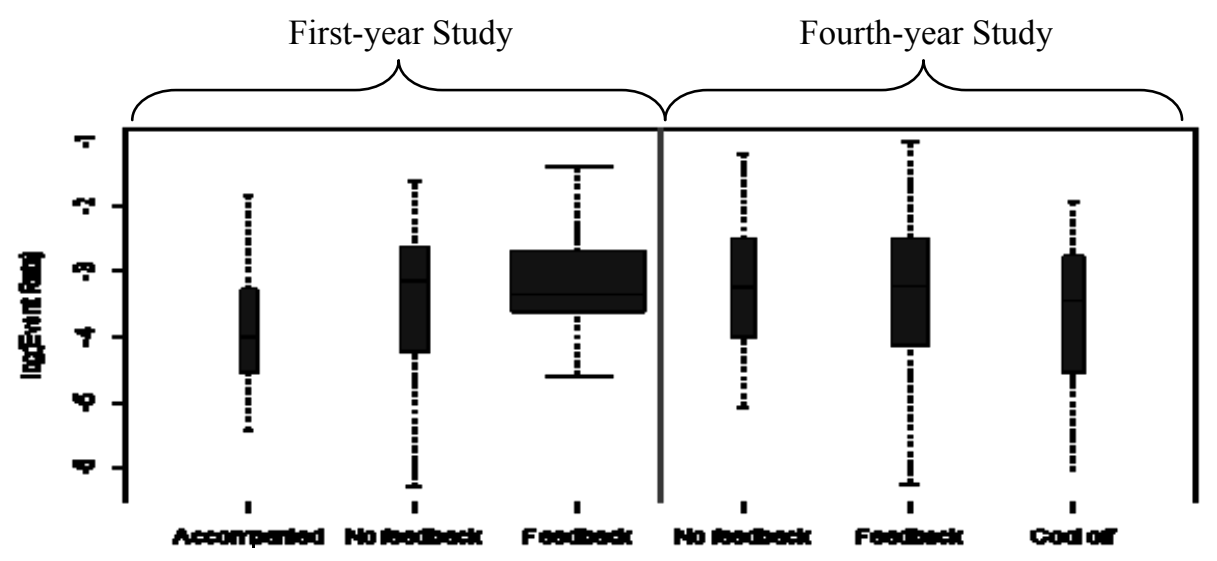

Figure 1. Events rates by study stages

Repeated measures ANOVA over the fourth-year stages suggested that events rates may not be similar $\left(\mathrm{F}_{(2,52)}=6.42, \mathrm{p}\right.$. value $\left.=0.003\right)$. The Tukey post-hoc test $(\alpha=0.05)$ could not indicate a 
partition of the stages for two or three groups. Yet a paired $t$ test analysis suggested that the log events rate in the cool-off stage was significantly lower compared to both the non-feedback stage (paired t. value $=3.06$, p. value $=0.004$ ) and the feedback stage (paired t. value $=2.38, \mathrm{p}$. value $=$ $0.024)$. The events rates in the feedback stage were lower than in the non-feedback stage.

However, these differences are not statistically significant (paired t. value $=1.75$, p. value $=0.09$ ). The difference between the cool-off stage and the previous stages can be considered minor in comparison to the difference between the accompanied stage and other stages. This may be the reason for the formal post-hoc test results. No significant difference (paired t . value $=0.39, \mathrm{p}$. value $=0.70$ ) was found between the events rates in the fourth-year study and those in the firstyear study (excluding the accompanied phase).

\section{Temporal Effects on Events Rates}

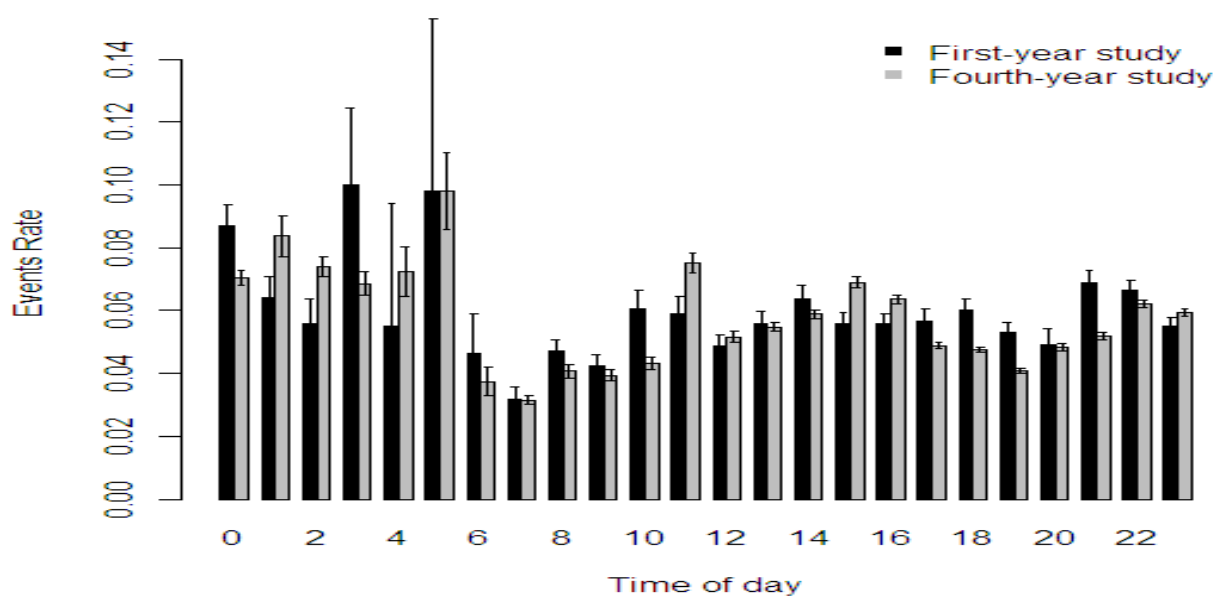

Figure 2. Events rates by the time of day

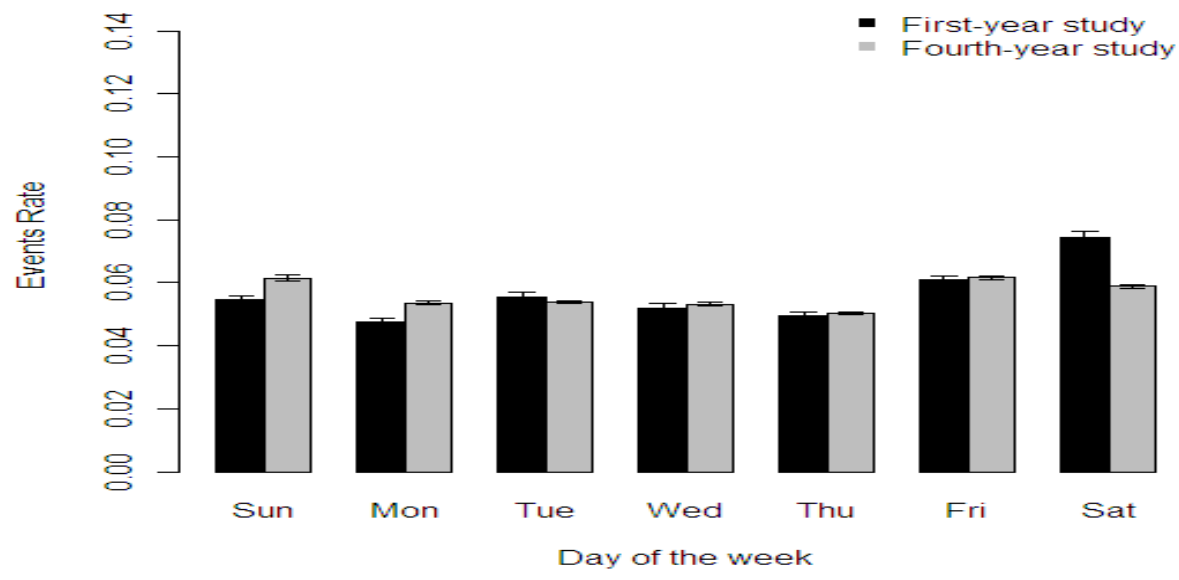

Figure 3. Events rates by the day of the week

Figure 2Figures 23 present the events rates as a function of the time of day and day of week for both studies. The results show higher events rates at night, from midnight to 6:00, and during the weekend. These results are consistent with young drivers' safety literature, which repeatedly 
reports increased involvement in crashes during weekends and at night (See for example, Åkerstedt and Kecklund, 2001; Doherty et al., 1998; Musicant et al., 2010).
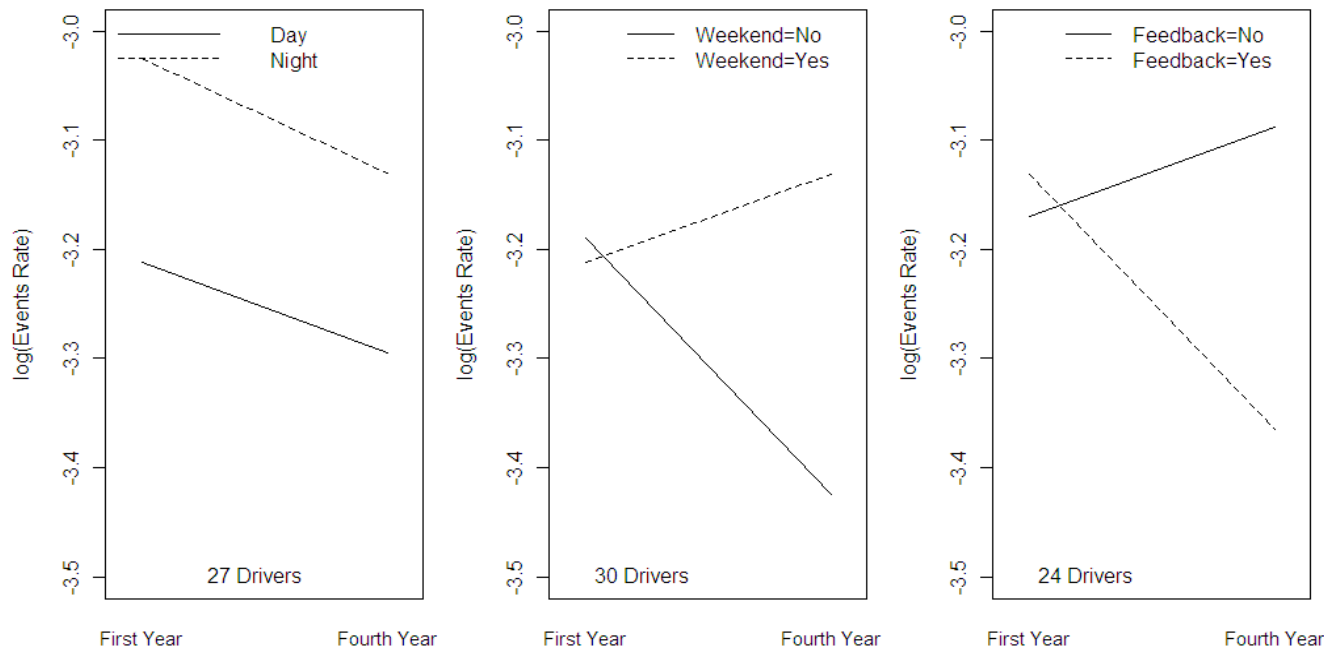

Figure 4. Events rate by night, weekend and feedback

Figure 4 presents the comparison of the events rates in the two studies by time of day (left), by day of week (centre) and by feedback provision (right). This information is presented only for drivers with trips in all combinations. Since the drivers had repeated measures for both factors described in each subplot, a 2X2 design with repeated measures was implemented to test significance. The effect of night-time on the events rate is prominent in both studies (t. value $=$ 2.08 , p. value $=0.04$ ). Also significant is the effect of the interaction between weekend and year (first or fourth), where the events rate for more experienced drivers is higher on weekends (t.value $=9.25$, p. value $<0.001$ ). The effect of feedback is also notable, as its provision in the fourth year (interaction) causes a decreased events rate $(\mathrm{t}$. value $=2.12$, $\mathrm{p}$. value $=0.03)$. The effect of feedback and of the weekend in the fourth year was further explored.

Figure 5 (below) depicts the mean over the events rate for a subset of 21 drivers with observations at each of the combinations (to create balanced data). The figure suggests that the transition to feedback has a noticeable effect in situations where the events frequency is relatively high (as in the weekend), and that driving with IVDR may trigger improvement in driving behavior. 


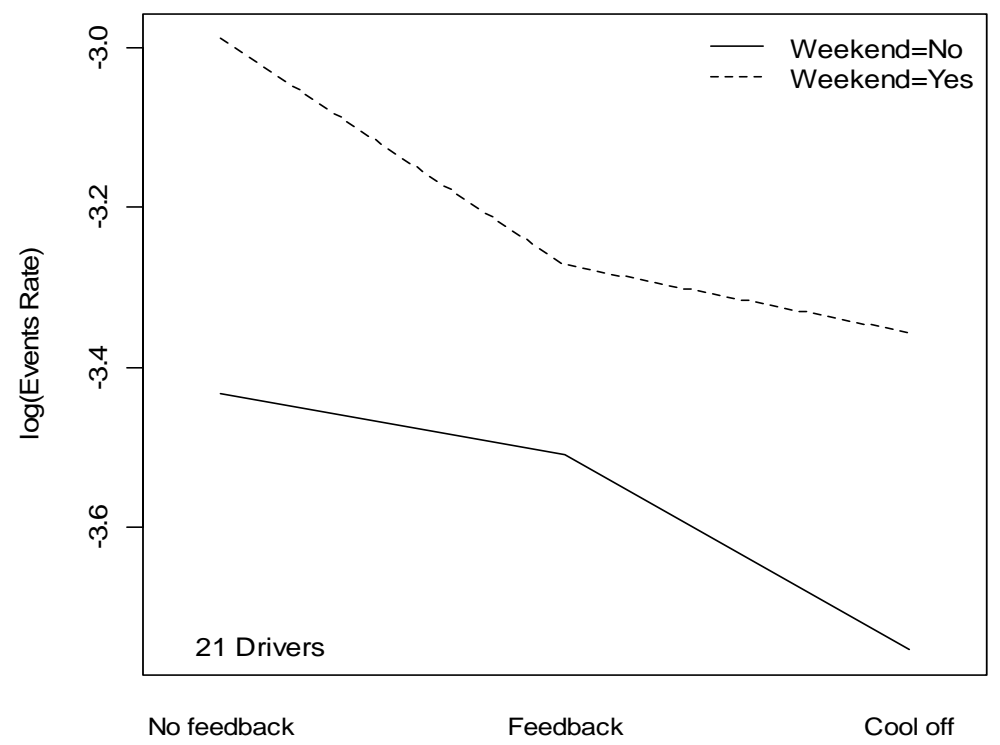

Figure 5. Interaction plot in the fourth-year study for study stage and weekend effects

\section{CONCLUSION}

This paper presents an attempt to analyse and compare, based on IVDR, the driving of young drivers with 3-4 years of experience to the behaviour of the same individuals in the first year immediately after licensure.

When considering the results, it is important to take into account that the analyses are based on a relatively small sample. The sample is probably biased towards individuals that have high awareness of, and positive attitudes towards, traffic safety, as indicated by their willingness to volunteer for the study. In addition, the status of the participants - a large fraction of them in military service - influences their exposure patterns in a way that may be unique to this situation.

The comparison revealed that, in general, driving patterns remain similar. In the main, no significant change occurred in the events rates. However, the difference in risky behaviour between weekdays and weekends was more prominent in the fourth year than in the first year, and an interesting improvement in driving behavior occurred during the cool-off stage. This may suggest that young experienced drivers improve their safety level while driving with IVDR. In addition, our results indicate that feedback does have a significant impact on the driving behavior of young experienced drivers.

As discussed earlier, the results should be considered with respect to the sample's characteristics. However, the data collection methodology and the way the data was analysed are more robust. The analysis results obtained should be considered an example of the potential of what may be done with this kind of data; IVDR technology is a valuable tool for understanding driving patterns and behavior. The detailed analysis of events provides insights into safety issues and should be further used to evaluate driving behavior and safety issues. 


\section{REFERENCES}

Åkerstedt, T. and Kecklund, G. (2001). Age, gender and early morning highway accidents. European Sleep Research Society, Vol. 10, pp.105-110.

Dingus T.A., Klauer S.G., Neale V.L., Petersen A., Lee S.E., Sudweeks J., Perez M.A., Hankey J., Ramsey D., Gupta S., Bucher C., Doerzaph Z.R., Jermeland J. and Knipling R.R. (2006). The 100-car naturalistic driving study phase II - results of the 100-car field experiment, Report DOT-HS-810-593, Department of Transportation, Washington DC.

Doherty, S.T., Andrey, J.C. and MacGegor, C. (1998). The situational risk of young drivers: the influence of passengers, time of the day and day of week on accident rates. Accident Analysis and Prevention, 30, pp.22-45.

Lotan, T. and Toledo, T. (2007). Driving patterns of young drivers within a driver licensing system. Compendium of Papers of the 86th Annual Meeting, Transportation Research Board, Washington DC, USA.

Musicant, O., Bar-Gera H. and Schechtman, E. (2010). Electronic records of undesirable driving events. Transportation Research F. Vol 13, Iss.2 pp.71-79.

Musicant, O. and Lampel, L. (2010). When Technology Tells Novice Drivers How to Drive. Journal of Transportation Research Record. Issue No. 2182, pp.8-15.

Prato, C.G., Toledo, T. Lotan. T. and Taubman-Ben-Ari, O. Modeling the behavior of novice young drivers during the first year after licensure (2010). Accident Analysis and Prevention. Vol 42, Iss. 2 pp. 480-486.

Toledo, T., Musicant, O. and Lotan, T. (2008). In-vehicle data recorders for monitoring and feedback on drivers' behavior. Transportation Research C 16, pp. 320-331.

Williams, A.F. (2003). Teenage drivers: patterns of risk. Journal of Safety Research, 24, pp.5-15. 